\title{
DEPENDENCE OF CREEP FAILURE PROBABILITY ON THE SIZE OF METALLIC SPECIMENS
}

\author{
Krzysztof NOWAK \\ *Faculty of Civil Engineering, Strength of Material Department, Cracow University of Technology, ul. Warszawska 24, 31-155 Kraków \\ kn@limba.wil.pk.edu.pl

\begin{abstract}
The occurrence of statistical size effect is considered for damage in creep conditions. The numerical and experimental analysis have been performed. The obtained results are ambiguous. Numerical models confirm the scale effect which can be statistical or deterministic one. But this effect has no experimental verification. It may suggest that the weakest link model cannot be applied in creep conditions. Explanation of this needs further investigations.
\end{abstract}

Key words: Size Effect, Creep Damage, Cellular Automata

\section{INTRODUCTION}

As is well-known, the specimen or structural element size influences the value of fatigue strength (Kocańda and Szala, 1991). When the size of element is larger the strength of specimen is lower. This phenomenon, known as size effect, can be described by probabilistic Weibull model (1939). The damage in creep conditions has many common aspects to fatigue failure. So it can be expected that creep strength dependence upon the specimen size is similar. But there are not so much papers describing size effect or lack of it for creep (e.g. Yatomi et al., 2003) so, evidence of this is not obvious.

\section{THE STATISTICAL AND DETERMINISTIC SIZE EFFECT}

The size effect described by Weibull is called statistical, as its nature is connected with statistical dispersion of material properties. This effect can be modelled by the so-called weakest link model (see Fig. 1). It assumes that specimen is made of many chains of random strength. The chains are linked in series, and therefore the strength of the whole specimen is determined by the weakest link. The longer specimen is, the probability of lower strength is larger.

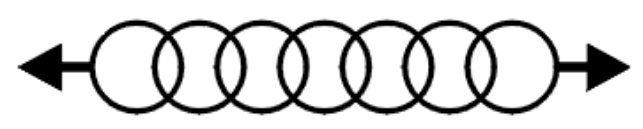

Fig. 1. The weakest link model

Any characteristic dimensions do not occur in the statistical size effect. It is caused mainly by nonhomogeneity of material or variability of specimen cross-section. The serial connections of chains correspond to situation when occurrence of first defect in specimen cause all the specimen to failure. In case of fatigue it is often related to existence of microcracks or microdefects, where the fatigue failure can arise. For larger specimen the prob- ability of existence of critical defect is larger and the failure can occur at lower number of cycles (Carpinteri et al, 2002, 2004, 2009, 2010).

In statistical size effect the power law is used for description of dependence of element size:

$$
\mathrm{f}(D)=c_{0} D^{s}
$$

where $f(D)$ is a function describing examined parameter (e.g. strength), $D$ is the structural element size, and $c_{0}, s$ are material constants. The power law is the only one, which allows us to describe size dependency without any characteristic length (Bažant, 1999).

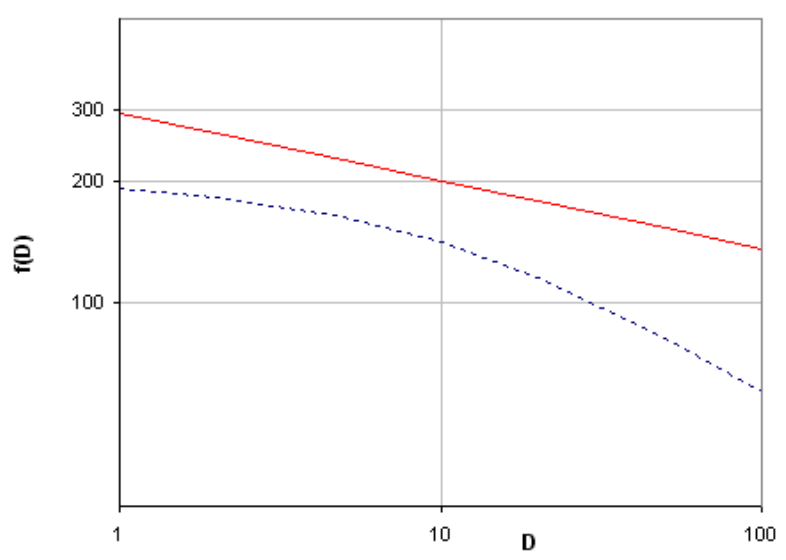

Fig. 2. Comparison of statistical and deterministic size effects. Solid red line - statistical size effect, eq. (1) for $s=-1 / 6$, dashed blue line deterministic size effect, eq. (2)

In contradiction to statistical size effect the characteristic length can occur in deterministic size effect. In this case not only statistical dispersion, but also relation of specimen size to some characteristic material dimension, is responsible for size effect. It makes the size effect more deterministic. This phenomenon is well described for materials with large inhomogeneities like 
concrete, rocks. Exemplary law for such effect can be stated in form of equation (Bažant, 1984):

$$
\mathrm{f}(D)=f_{0}\left(1+\frac{D}{D_{0}}\right)^{-1 / 2}
$$

where $D_{0}$ is characteristic dimension and $f_{0}$ material constant Comparison of statistical and deterministic size effect laws is shown in Fig. 2.

It can be shown for the weakest link model that the mean value of nominal strength is (Bažant, 1999):

$$
\bar{\sigma}=s_{0} \Gamma\left(1+m^{-1}\right)\left(\frac{V_{0}}{V}\right)^{1 / m}
$$

and the coefficient of variation:

$$
V=\sqrt{\frac{\Gamma\left(1+2 m^{-1}\right)}{\Gamma\left(1+m^{-1}\right)}-1}
$$

where $m$ is the Weibull modulus, $s_{0}$ is a material parameter, $V$ is the examined volume of material, $V_{0}$ is the volume of the smallest possible test specimen, $\Gamma$ - is the gamma function. In equation (4) the value of the coefficient of variation depends only on modulus $m$, so it is often used to determine this parameter. It usually varies from 5 to 50 . The small value of $m$ gives strong dependency upon the specimen dimensions and also large value of coefficient of variation. When the scatter, represented by coefficient of variation, of any variable is known it is possible to estimate the sensitivity of this variable to size effect. Not always the value of the modulus $m$ determined from equation (4) corresponds to the mean value determined from equation (3). It means that the deterministic size effect exists, what is characteristic for concrete or other quasi-brittle materials (Bažant, 1999).

\section{RANDOMNESS OF CREEP FAILURE}

The phenomenon of creep is characterised by large randomness. It causes that the scatter of basic parameters describing creep failure (time to failure, strain at failure) is significant. It is caused mainly by not identical conditions of creep tests (variations of temperature, eccentricity, see Hayhurst, 1974). But even ensuring special accuracy in test performance, the scatter of results is significant (e.g. Farris et al., 1990, obtained scatter of times to failure in test of copper with coefficient of variation about $60 \%$ ). This variability of the results can be explained by nonhomonegeity of material connected with its microstructure.

Estimation of Weiibull modulus from equation (4) by fitting to coefficient of variation equal to $60 \%$ gives $m=1.7$. This value defines very strong dependency on specimen size, e.g. two times larger specimen should give reduction of creep resistance of about $33 \%$. Existing experimental data shows that there is no size effect for creep of metallic materials or it is very small. E.g. Yatomi et al. (2003) made comparison of creep crack growth for welds of sizes: 15, 25 and $50 \mathrm{~mm}$ and claimed no size effect.

\section{TIN WIRE EXPERIMENTS}

The creep experiments are very time consuming. Therefore there is not much data allowing to draw conclusions about proba- bilistic behaviour of material, i.e. the nature of scale effect. This is the reason why own experiments were performed for wire made of tin alloy Sn60Pb40, which has small melting temperature $(460 \mathrm{~K})$ and creeps in room temperature. The specimens have $1 \mathrm{~mm}$ of diameter and were loaded by constant force giving nominal stress $11.8 \mathrm{MPa}$. Two lengths of specimens were examined: short - $54 \mathrm{~mm}$, and long $-93 \mathrm{~mm}$. The obtained times to failure are shown in Table 1.

Tab. 1. Experimental results for tin alloy wire

\begin{tabular}{|c|c|c|c|}
\hline $\begin{array}{c}\text { length } \\
\text { of specimen } \\
\text { [mm] }\end{array}$ & $\begin{array}{c}\text { number } \\
\text { of probes }\end{array}$ & $\begin{array}{c}\text { mean time } \\
\text { to failure [s] }\end{array}$ & $\begin{array}{c}\text { coefficient } \\
\text { of variation }\end{array}$ \\
\hline 54 & 14 & 1617.3 & $15.6 \%$ \\
\hline 93 & 11 & 1777.3 & $20.8 \%$ \\
\hline
\end{tabular}

The observed in experiment scale effect is reverse to the expected one. The mean time to failure for longer specimens is about $10 \%$ greater than for shorter ones. The explanation of this phenomenon requires much more experiments to perform to exclude any accidental cause of this behaviour.

\section{NUMERICAL ANALYSIS}

\subsection{FEM model}

In order to numerically verify statistical size effect, series of simulations were performed by author. Rectangular specimen $5 \times 2.5 \mathrm{~mm}$ was modelled in plane strain state by the finite element method. ABAQUS code was used. Keeping constant mesh density the simulations were performed for lengths: 10, 20, 40, 80 and $160 \mathrm{~mm}$. The specimen of $20 \mathrm{~mm}$ was chosen as a reference one. The material model was Hook-Norton-Kachanov (equations after Bodnar and Chrzanowski, 2002):

$$
\begin{aligned}
& \varepsilon_{i j}=\varepsilon_{i j}^{e}+\varepsilon_{i j}^{c} \\
& \varepsilon_{i j}^{e}=D_{i j k l}^{-1} \sigma_{k l} \\
& \frac{\partial \varepsilon_{i j}^{c}}{\partial t}=B\left(\frac{\sigma_{e f f}}{1-\omega}\right)^{n} \frac{\partial \sigma_{e f f}}{\partial \sigma_{i j}} \\
& \frac{\partial \omega}{\partial t}=A\left(\sigma_{e q}\right)^{\mu}
\end{aligned}
$$

$\sigma_{e q}=\alpha \frac{\sigma_{\max }}{1-\omega}+(1-\alpha) \frac{\sigma_{e f f}}{1-\omega}$

where: $\varepsilon_{i j}, \varepsilon_{i j}^{e}, \varepsilon_{i j}^{c}-$ total, elastic and creep strain tensors, respectively, $\sigma_{k l}$ - stress tensor, $D_{i j k l}$ - elastic constants matrix, $B, n$, $A, m, \alpha$ - steady-state creep and damage material constants, $\sigma_{\text {max }}, \sigma_{\text {eff, }} \sigma_{\text {eq }}-$ main positive principal stress, Huber-Mises effective stress and equivalent stress, respectively, $\omega-$ scalar damage parameter $(0<\omega<1), t-$ time.

The material constants were fitted to data for copper in temperature $723 \mathrm{~K}$ and mean grain diameter 30 microns obtained by Feltham and Meakin (1959): elastic constants - Young modulus 82.7GPa, Poisson ratio 0.33 , creep constants $-B=1.18 \mathrm{MPa}^{-n} \mathrm{~s}^{-1}$, 
$n=4.17$ damage constants $-A=5.04 \mathrm{MPa}^{-\mu} \mathrm{s}^{-1}, \mu=2.87, \alpha=1$. In order to ensure randomness of results, the random initial damage parameter was introduced. Its values were spread over a volume of specimen according to exponential distribution with expected value $\omega_{0}=0.006$.

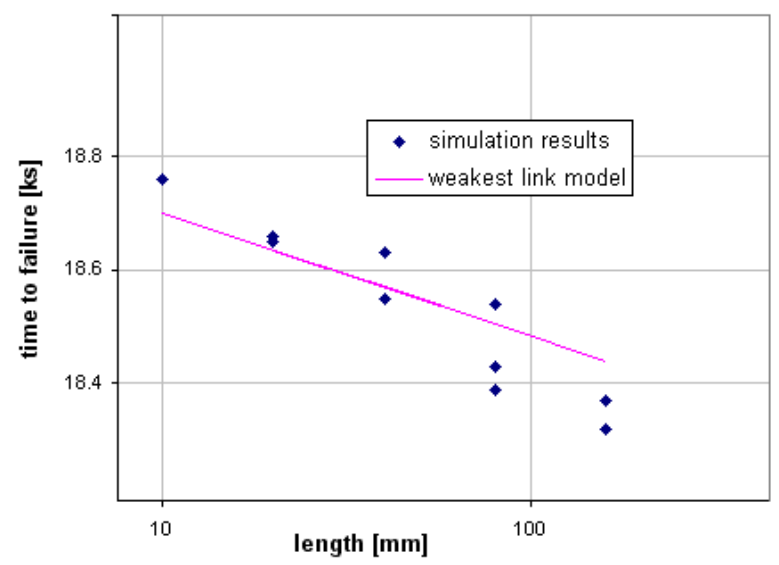

Fig. 3. Size dependency of time to failure obtained from numerical simulation in comparison with the weakest link model - eq. (6)

The scatter of creep test results in FEM model was obtained from 8 probes performed for reference size of specimen. Every probe had unique random configuration of initial damage parameter. For uniaxial tensile loading corresponding to equivalent stress $\sigma_{\text {eq }}=34.6 \mathrm{MPa}$, the obtained scatter was very small. The mean value of time to failure was $18635 \mathrm{~s}$ and coefficient of variation was $0.65 \%$. This coefficient corresponds to Weibull modulus $m=196$ according to equation (4). Despite the relation is very weak it is possible to compare the values resulting from the weakest link model with results of numerical simulations (see Fig. 3). According to the weakest link model and the Monkman-Grant relation (1956), the size dependency of time to failure can be calculated as follows:

$t_{f}=t_{f 0}\left(\frac{l_{0}}{l}\right)^{1 / m}$

where: I is length of specimen, 10 is length of reference specimen, $t_{f o}$ is value of time to failure for reference size. The correlation of simulation results with the model is quite good, although the size effect is very weak due to uniform material properties over the volume of specimen. The influence of characteristic size, which in this case is finite element size, is also very small. Therefore it can be stated that the numerical model confirms the statistical size effect.

\subsection{Multiscale model}

The second model analysed is the multiscale CAFE model (see Nowak, 2011). It allows to introduce nonhomogeneity of microstructure of material resulting in large scatter of simulation results. The model consists of two main parts describing deformation and damage. They operate on different scales and use different methods of analysis.

The deformation is modelled on macro level using discretised version of constitutive equation (7):

$\dot{\varepsilon}_{c}=B_{1} \varepsilon_{c}^{-2}\left(\frac{\sigma}{1-\omega}\right)^{n_{1}}$

where $n_{1}, B_{1}$ are creep constants fitted to primary creep behaviour according to Chrzanowski's (1972) proposal of description of primary, secondary and tertiary creep.

The damage development processes is modelled by Cellular Automata (CA) technique (see Raabe, 2002, for review). It operates on microstructure of polycrystalline material using Representative Volume Element (RVE) approach. The CA model reflects the discrete nature of material. The state value of a single cell and its neighbourhood allows to distinguish if cell belongs to grain interior or grain boundary, it also allows to model voids or cracks. Also the randomness of creep process can be modelled by $\mathrm{CA}$ very easily by introduction the random microstructure and by random process of damage development. The algorithm uses in current simulation prefer development of intergranular cracks, as size effect is more significant in brittle failure. The relative length of longest crack inside the RVE is used as damage parameter $\omega$ in deformation equation (7). In turn the deformation obtained by FE model influences the current size of RVE. It results in mutual coupling of both scales.

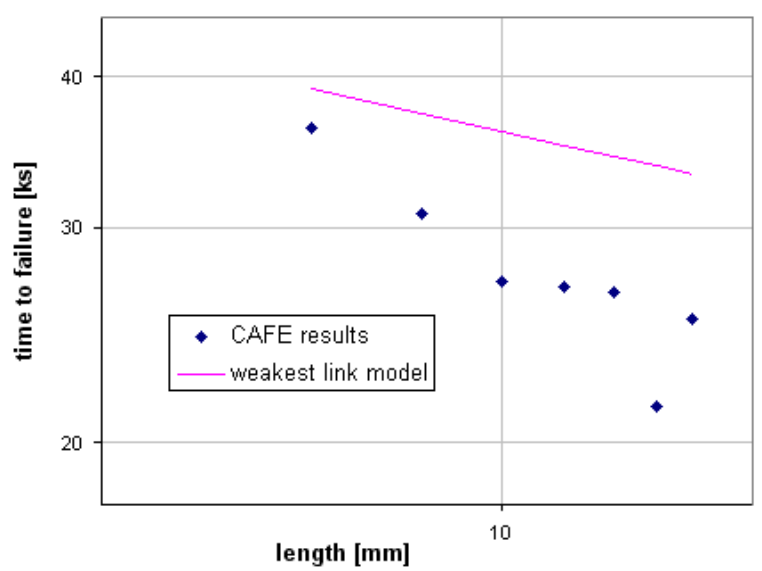

Fig. 4. Size dependency of time to failure obtained from CAFE simulation in comparison with the weakest link model

Similar to the previous case, the simulations were performed for specimen of $5 \mathrm{~mm}$ width and different lengths from 5 to $20 \mathrm{~mm}$, keeping constant the density of FE mesh and size of RVE (in cells). The material parameters were also fitted to results of experiment for copper by Feltham and Meakin (1959) but the loading in uniaxial tensile test was $\sigma_{\text {eq }}=30.0 \mathrm{MPa}$. The reference length was $5 \mathrm{~mm}$. The scatter of results was produced by different initial microstructure of grains of mean diameter about 100 cells, where RVE size was 320 cells. For 8 tests of reference length size the mean value of time to failure was 39100 s with coefficient of variation equals to $14.1 \%$. This scatter is comparable with scatter of experiments results (cf. Garofalo et al., 1961, and Tab. 1). According to equation (4) corresponding Weibull modulus is $m=8.5$. Comparison of the simulations results for different specimen length with values predicted by equation (3) gives much strongest relation with scale (see Fig. 4). This indicates that this size effect cannot be considered as only statistical one. Existence 
of at least two characteristic dimensions like grain size and RVE size results in deterministic size effect. The effect is strong as they are quite close one to another.

\section{CONCLUSIONS}

It has been shown that the numerical simulations performed by the author can be used in the description of scale effect in creep tests.

The scatter of results obtained by means of multiscale model coincides with scatter of times to failure obtained from experiments. This scatter should cause statistical scale effect described by weakest link model. However, the results of own and also other experiments do not show such effect. The reason of that is not clear. The explanation of that phenomenon requires more experimental and numerical investigations.

\section{REFERENCES}

1. Bažant Z.P. (1984), Size Effect in Blunt Fracture: Concrete, Rock, Metal, J. Eng. Mech., 110, 518-535.

2. Bažant Z.P. (1999), Size effect on structural strength: a review, Archive of Applied Mechanics, 69, 703-725.

3. Bodnar, A., Chrzanowski, M. (2002), On creep rupture of rectangular plates, ZAMM, 82, 201-205.

4. Carpinteri A., Spagnoli A. (2004), A fractal analysis of size effect on fatigue crack growth, Int. J. of Fatigue, 26, 125-133.

5. Carpinteri A., Spagnoli A., Vantadori S. (2002), An approach to size effect in fatigue of metals using fractal theories, Fatigue Fract. Engng. Mater. Struct., 25, 619-627.

6. Carpinteri A., Spagnoli A., Vantadori S. (2009), Size effect in S-N curves: A fractal approach to finite-life fatigue strength, Int. J. of Fatigue, 31, 927-933.
7. Carpinteri A., Spagnoli A., Vantadori S. (2010), A multifractal analysis of fatigue crack growth and its application to concrete, Eng. Fract. Mech., 77, 974-984.

8. Chrzanowski M. (1972), On the Possibility of Describing the Complete Process of Metallic Creep, Bull. Ac. Pol. Sc. Ser. Sc. Techn., XX, 75-81.

9. Farris J.P., Lee J. D., Harlow D. G., Delph T.J. (1990), On the scatter in creep rupture times, Metallurgical and Materials Transactions, 21A, 345-352.

10. Feltham P., Meakin J.D. (1959), Creep in Face-Centred Cubic Metals with Special Reference to Copper, Acta Metallurgica, 7, 614627.

11. Garofalo F., Whitmore R.W., Domis W.F., Gemmingen F. (1961), Creep and creep-rupture relationships in an austenitic stainless steel, Trans. Metall. Soc. AIME, 221, 310-319.

12. Hayhurst D.R. (1974), The effects of test variables on scatter in high-temperature tensile creep-rupture data, International Journal of Mechanical Sciences, 16, 829-841.

13. Kocańda S., Szala J. (1991), Podstawy obliczeń zmęczeniowych, PWN, Warszawa (in Polish).

14. Monkman F.C., Grant N.J. (1956), An Empirical Relationship between Rupture Life and Minimum Creep Rate in Creep-Rupture Test, Proc. ASTM, 56, 593-620.

15. Nowak K. (2011), Uncertainty of lifetime for CAFE creep damage model, Computer Methods in Materials Science, 11, 315-323.

16. Raabe D. (2002), Cellular Automata in Materials Science with Particular Reference to Recrystallization Simulation, Ann. Review of Materials Research, 32, 53-76.

17. Weibull W. (1939), The Phenomenon of Rupture in Solids, Proceedings of Royal Swedish Institute for Engineering Research, 153, 5-55.

18. Yatomi M., Nikbin K.M., O'Dowd N.P. (2003), Creep crack growth prediction using a damage based approach, International Journal of Pressure Vessels and Piping, 80, 573-583. 